\title{
Performance of 2D SOVA Along and Across Track in Shingled Magnetic Recording Media
}

\author{
Muhammad B. Abdulrazaq, Mohammed Z. Ahmed, and Paul Davey
}

\begin{abstract}
Serial Concatenation of Two Dimensional Soft Output Viterbi Algorithm (2D-SOVA) and regular Viterbi Algorithm (VA) for 2D equalisation and detection of Shingled Magnetic Recording (SMR) media provides excellent performance as compared to the use of 1 Dimensional (1D) maximum likelihood detector. In this paper, we implement and evaluate the performances of two versions of it. The first version performs 2D SOVA along the tracks to eliminate the effect of inter-symbol interference (ISI) and then the Viterbi detector across the tracks to remove inter-track interference (ITI). The second version carries out 2D-SOVA across the tracks and VA along the tracks. The results for high ITI and ISI show a better performance when using 2D-SOVA across the track with a small difference in computational complexity in favour of 2D-SOVA across the tracks.
\end{abstract}

Keywords - 2D detection, Maximum Likelihood Detection, Shingled Magnetic Recording, SMR, Soft Output Viterbi Algorithm, SOVA.

\section{INTRODUCTION}

$\mathrm{T}$ HE capacity of magnetic recording media enjoyed a steady increase, until recently when the increase slowed down due to approaching the Super-Paramagnetic limit of the magnetic recording media [1]. In an attempt to keep the trend of the increase going on, some solutions are proposed. Among the popular are Bit Patterned Magnetic Recording (BPMR), Energy Assisted Magnetic Recording (EAMR) and Shingled Magnetic Recording (SMR) [2].

EAMR uses energy to soften the media for writing, therefore, it usually requires extra energy for its running. In addition to having a very cumbersome write head, its energy consumption is not suitable for today's low-power devices, especially mobile devices. BPMR requires the creation of magnetic islands on the media to reduce interference. This totally new media design has susceptibilities to shift in the position of the head and needs a radical change in media

Paper received November 9, 2016; revised March 2, 2017; accepted March 3, 2017. Date of publication July 31, 2017. The associate editor coordinating the review of this manuscript and approving it for publication was Prof. Branimir Reljin.

This paper is a revised and expanded version of the paper presented at the 23rd Telecommunications Forum TELFOR 2015 [8].

Muhammad Bashir Abdulrazaq works with Department of Electrical and Computer Engineering Ahmadu Bello University Zaria, Nigeria and currently doing his PhD at Plymouth University U.K (e-mail: muhammad.abdulrazaq@plymouth.ac.uk).

Mohammed Ahmed Zaki is an associate professor at School of Electronics, Mathematics and Computing, Plymouth University, UK (e-mail: m.ahmed@plymouth.ac.uk ).

Paul Davey is an associate professor at School of Electronics, Mathematics and Computing, Plymouth University, UK (e-mail: p.davey@plymouth.ac.uk ). design [3]; SMR does not change the media design or need a radically different head. It is based on overlapping written data so as to eliminate the write head limitation of bit size and make the tracks as small as possible. The success of this technique lies with a proper signal processing and dataupdate approach [4].

Because in SMR the tracks are very closely packed, to increase areal density, the problem of Inter-Track Interference (ITI) becomes very significant and therefore has to be dealt with to get an adequately performing SMR disk [2]. This is in addition to Inter-Symbol Interference (ISI) along the tracks, which makes the problem a two dimensional (2D) problem.

The read head size is instrumental in the amount of signal energy collected, therefore rather than reducing the size of the read head, to avoid the ITI, DSP techniques for utilising or removing the ITI are explored by researchers [5].

Some techniques explored include: using a linear equaliser to cancel ITI in one direction and Partial Response Maximum Likelihood (PRML) to detect the shaped signal; using a linear Equaliser to cancel ISI along tracks and a Maximum Likelihood (ML) detector to detect the signal [6]; or using a full 2D SOVA [7] [8]. The most important advantage of using linear equalisers is to reduce complexity, even though it is not optimal especially under high ISI or high ITI. Full 2D SOVA detection has a good performance but is very complex compared to the use of linear equalisers.

In the past, Full 2D SOVA has not received much attention. But recently due to anticipated development in IC technology, it has been receiving attention [7] [9]. In this paper, we present two implementations of serial concatenation of 2D-SOVA with the Viterbi Detector for Two Dimensional equalisation of SMR media. One implementation uses 2D-SOVA along the track to remove the effect of ISI and the Viterbi detector across-tracks to remove the effect of ITI (Along-Track 2D-SOVA). The second implementation uses 2D-SOVA across the tracks to remove the effect of ITI and then the Viterbi Detector along the tracks to remove the effect of ISI (Across-Track 2DSOVA). Their performances and complexities are compared and discussed.

The rest of this paper is organised as follows. Section II gives an overview of the channel model, shaping equaliser and 2D SOVA detection; section III elaborates on the modelling and design of the detectors; section IV evaluates the features of the two detectors used in getting the results; section $\mathrm{V}$ presents some results from the designs and their comparisons, and section VI presents conclusions drawn from the results. 


\section{OVERVIEW OF CHANNEL AND EQUALISATION}

\section{A. PMR Channel}

The read head (Magneto Resistive (MR) read head in this case) of Perpendicular Magnetic Recording (PMR) media picks the magnetisation of a point on the media a distance away before reaching or after passing the bit position. This creates Inter-Symbol Interference (ISI) among neighbouring bits. The response can be modelled by convolution of stored data with isolated response (Equation (1)) as shown in equation (2) [10]. This equation is the first order approximation of the response.

$$
\begin{gathered}
s(t)=V_{\max } \tanh \left(\frac{2 t}{0.579 \pi T_{50}}\right) \\
z(t)=\sum_{i} x_{i} h(t-i B)+\sum_{i} d_{i} a_{i} s^{\prime}(t-i B)
\end{gathered}
$$

where, $V_{\max }$ is the maximum amplitude of single bit transition, $T_{50}$ is the time taken for voltage of single transition to change from $-V_{\max } / 2$ to $V_{\max } / 2$, " $t$ " is the sampling time, " $i$ " is the position of an interfering bit with respect to the central bit, " $B$ "' is the bit period, $x_{i}$ is the actual written data, $d_{i}=\left(x_{i}-x_{(i-1)}\right) / 2$, " $a_{i}$ " is the standard deviation of jitter noise, " $s$ " " is the differential of equation (1), and

$$
h(t-i B)=(s(t-i B)-s(t-i(B+1))) / 2
$$

In SMR media the presence of closely packed tracks means the read head may pick information from side tracks. Therefore, the total read signal is the sum of the contributions of signals from all interfering tracks and other noises $\left(n_{i, j}\right)$ as shown in equation (4). Where " $j$ " ranges across all interfering tracks and " $w_{j}$ " is the fractional contribution of each track.

$$
y_{i, j}=\sum_{i} \sum_{j} x_{i, j} h_{i} w_{j}+\sum_{i} \sum_{j} d_{i, j} a_{i, j} s_{i}^{\prime} w_{j}+n_{i . j}
$$

The variables " $h$ " and " $s_{i}^{\prime}{ }_{i}$ " are used in place of $h(t-i B)$ and $s^{\prime}(t-i B)$ for easier writing of equation (4).

\section{B. Equalisation}

To reduce the complexity of the detection, the data is shaped to a target response. A "target response" is a desired interference pattern of a certain number of symbols. The number of symbols is termed the "target length". The target is normally represented as a vector (target vector) of coefficients of consecutive symbols of the data. A suitable target, which minimises the mean squared error, can be determined using procedures presented in [5]. For the method presented here, the best 1 dimensional target vector of length 3 found and used here is $[1,2,1][6]$.

$$
H=\left[\begin{array}{ccccc}
h_{n} & h_{n+1} & h_{n+2} & h_{n+3} & h_{n+4} \\
h_{n-1} & h_{n} & h_{n+1} & h_{n+2} & h_{n+3} \\
h_{n-2} & h_{n-1} & h_{n} & h_{n+1} & h_{n+2} \\
h_{n-3} & h_{n-2} & h_{n-1} & h_{n} & h_{n+1} \\
h_{n-4} & h_{n-3} & h_{n-2} & h_{n-1} & h_{n}
\end{array}\right]
$$

If equation (5) is formed from the channel response in equation (3), where " $h_{n}$ " is the coefficient of the bit with the highest amplitude, and a vector " $T$ " is formed by padding the target vector with zeros to make it the length of the shaping equaliser, then the equaliser coefficients can be found as shown in equation (6) [11].

$$
E=H^{-1} T
$$

\section{2D SOVA}

Multilevel Soft Output Viterbi Algorithm is a detection mechanism that carries out the Viterbi algorithm to produce an output that gives the level of reliability for a symbol that has more than two levels. Each state usually has more than 2 branches terminating at it or coming out of it.

In a $2 \mathrm{D}$ situation (such as SMR), a certain number $(T)$ of interfering bits in one direction are considered to be one symbol. Therefore, the number of levels of a symbol will be $\beta=2^{T}$. One less than the number of bits in the second direction is, therefore, the constraint length $(k)$. The number of states is determined from $2^{T^{*} k}$

For each state having $\beta$ branches terminating in it, $\beta$ Branch Metrics (BMs) are determined by adding the appropriate Distance Metric to the State Metric (SM) of the previous state from which it originates. The Euclidean distance of the received signal, from all the possibly sent signals, is used as the Distance Metric (See Equation (7)). The BMs for each state are compared and the one with the least distance is taken as the surviving SM to be used for a next symbol. In order to preserve the information of the complete symbol reliability, all the BMs can be stored in the history until a traceback is conducted to get the best symbol. This mode of saving history makes it possible to serially concatenate to other Maximum Likelihood (ML) detectors or decoders. The position of the surviving SM (Equivalent of Hard Decision) is also saved in a history to be used for traceback.

$$
D M_{t, n}=\left(y_{t}-x_{n}\right)^{2} .
$$

Periodically, trace back is carried out to determine the most probable symbol(s). The state with the best surviving metric is first determined to start the traceback from it. To reduce complexity, any state can be chosen if there is a very high probability of the states all merging to one path at the end of the traceback. Choosing any state to start from, makes the required Trace-Back length $(T B l=$ number of symbols kept in history) longer. The periodic Trace-Back can theoretically be as high as the $T B l$. This means " $T B l$ " number of symbols must be processed before a traceback is started, and the number of outputs determined will be the whole $T B l$. The period can be as low as one symbol. This means after every symbol is processed, a traceback will be conducted to get the output of one symbol. The Hard Decision History is used to identify from which state is the surviving metric chosen.

To avoid the task of frequent trace back in a situation with a very low trace back period, register exchange History update can be done. This is a situation where the history of the previous state, from which the surviving SM is chosen, is carried along to the new state. The history, therefore, represents the history of all the paths traversed, up to that state. In this situation, the history of the chosen state will just be lifted and used as output. The suitability of this method depends on the ease of moving the whole history which involves the use of lots of multiplexers. 


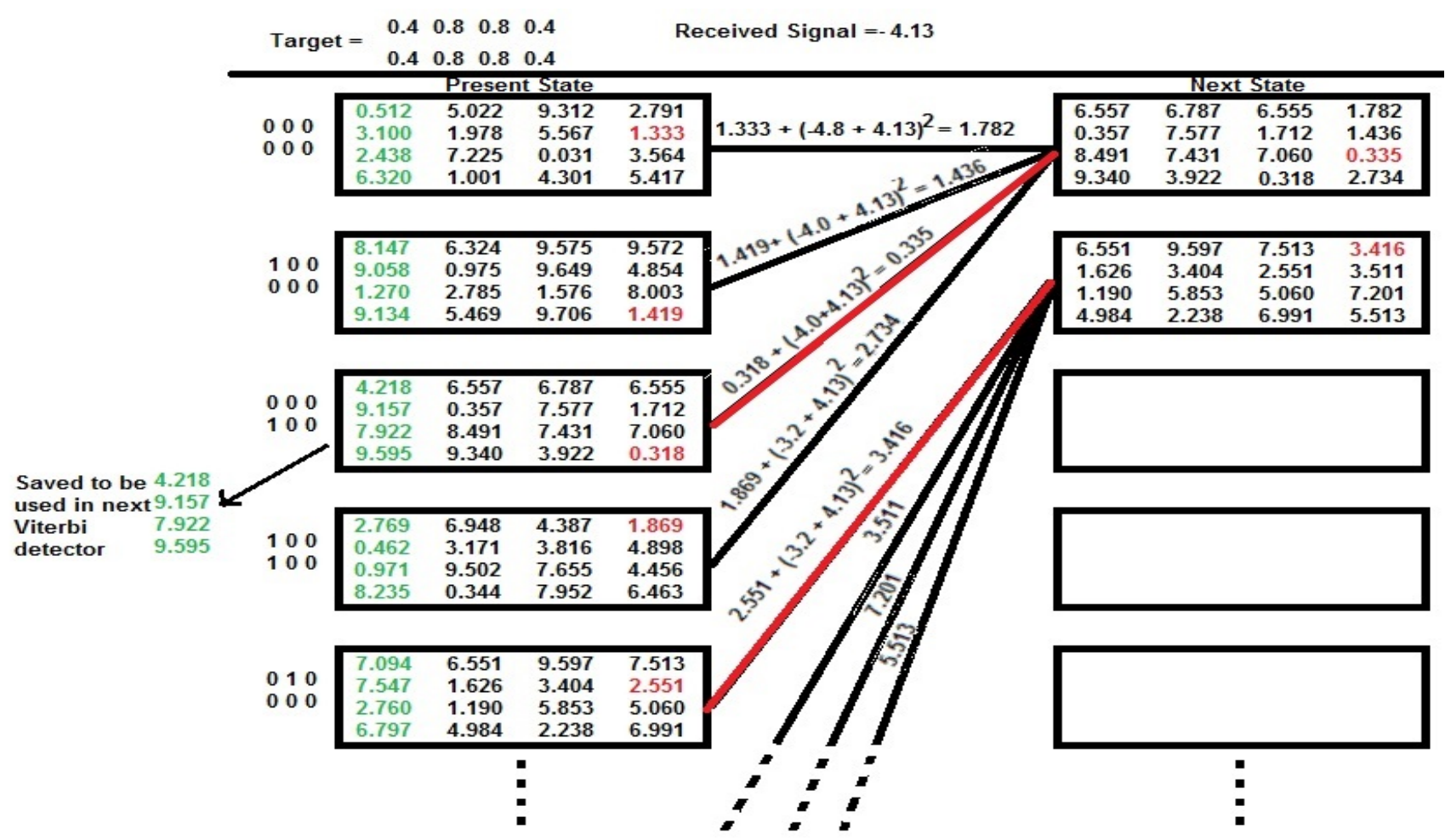

Fig. 1. 2D SOVA Trellis.

Fig. 1 shows a portion of 2D trellis for a two-track ITI system $(I T I=[1,1])$ with a target of length $4([0.4,0.8,0.8$, $0.4])$. Each block contains the history of a state. The data in red is the state-metric (SM), which is the minimum in the last column of each state. The minimum of these SMs is the state from which the first column (in green) is chosen to be saved as output. From the states that are visible (assuming the rest not shown have higher SMs) the third state has the minimum SM and therefore its green column will be saved as the output representing the likelihood of $[00,10,01,11]$ respectively. The $T B l$ in this example is 4 because we have 4 columns of BMs for each state.

The current received signal (-4.13 in the example) is used to find DM for each branch. It is then added to the SM of the given state from which it originates to get BMs, as shown in the branches in Fig. 1. The BMs are saved as the last (newest) column in a next state with their minimums as the new SM for each state. History of the state from which we obtained the new SM is then carried, discarding the green column, and appended to the BMs as the new history for that state. The process is repeated in the next cycle by using Next State data in place of Present State until the end of the data is reached. Regular normalisation should be done to avoid overflow of registers.

\section{A. Concatenated Viterbi Detector}

The BMs saved as output can now be used by another Viterbi detector as BMs. For a 4-level symbol, the four BMs selected will represent the distance metric of all the four possible symbols $(00,10,01,11)$ of the second Viterbi detector taken in the second direction.

\section{A. Channel Model}

The media is assumed to be written in a shingled form with 8 tracks of 4096 bits each ( $4 \mathrm{kB}$ per sector). Guard bands between sectors are assumed to have all zeros on them. This can be achieved by purposefully writing on the track before the sector, to separate the sectors. Because the last track will not be overwritten, it is assumed to be at least twice as wide as other tracks. ITI from two adjacent tracks is assumed (3-track ITI). Additive White Gaussian Noise (AWGN) contributes $20 \%$ of the noise while Jitter noise contributes the remaining $80 \%$. The signal to noise ratio $(\mathrm{SNR})$ is given as shown in equation (8)

$$
S N R=10 \log \left(\frac{V_{\max }^{2}}{\sigma_{w}^{2}+\sigma_{j}^{2}}\right) .
$$

where $\sigma_{w}$ and $\sigma_{j}$ are the standard deviation of AWGN and Jitter noise respectively.

\section{B. Shaping}

The received noisy data is first passed through a linear shaping equaliser that shapes the signal to a onedimensional target response along the track. Selection of target response suitable for this method of equalisation is similar to the method used in [6]. A target which produces equaliser that has the minimal noise amplification is chosen. Investigation shows that target $\left[\begin{array}{lll}11 & 20 & 11\end{array}\right]$ performs excellently for $T_{50} \geq 1.0$ [8]. But for hardware simplicity,

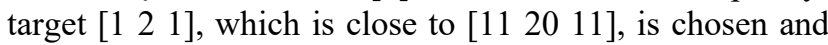
used for the results presented here. 


\section{SOVA Along-Track}

In this implementation, interfering bits from the two adjacent tracks together with the central track are assumed to be a single symbol $(T=3)$. This means the symbol will have 8 levels $\left(2^{T}\right)$. For a target length of 3 , constraint length $k=3-1=2$. Therefore, the number of states is $2^{2 * 3}=64$.

2D SOVA is used along the track, which produces 8 metrics that represent a 3-bit symbol (from adjacent bits of 3 tracks). These 8 metrics are used as the BMs for use in the Viterbi detector across the track that detects the final symbol from the interfering tracks. This means the BMs of the first symbol in the first track, the BMs of the first symbol in the second track and so on are assumed to be successive symbols on which the Viterbi detection is applied.

\section{SOVA Across-Track}

In this implementation, interfering bits along track (ISI) are considered to make one symbol. For a target length $3, T$ $=3$. Therefore, the number of levels per symbol is also 8 . Considering ITI from two adjacent tracks, the constraint length will also be 2 . This means the number of states is also 64.

2D SOVA is first used across tracks to remove the effect of ITI. This means the first symbols of all tracks are processed together, the second symbols of all tracks treated together and so on to the last positions. These produce 8 metrics per symbol position, which represent a symbol with the central bit and 2 bits interfering with it along the track. These 8 metrics are used as the DMs used in the Viterbi detector along the track that detects the final symbol from the interfering bits.

\section{Evaluation}

When applying 2D SOVA along a track, the data is directly passed into the shaping equaliser then immediately used for the 2D SOVA and the resulting 8 metrics also immediately used for the Viterbi detector. The next state metric of the Viterbi detector (4 of them) must be saved until adjacent bit on the next track is processed. This means a memory capable of storing 4 metrics for each of all symbols of a track is needed. In addition, history for the four possible states for the 8 tracks for all bits is also saved. The data will, at the end, be outputted in groups of 8 bits at a time across tracks. The latency of the system is the time delay between receiving the data and determining an output. In this situation, the latency that will be incurred comes from the delay produced by a shaping equaliser, delay for traceback $(T B l)$ of the 2D SOVA and other computational overhead from the Viterbi detection.

In the case of 2D SOVA across tracks, the received data is shaped then saved until at least the first symbol of the last track or all tracks is received. The shaped signal is then passed through a 2D SOVA across tracks. After any group of 8 symbols across track is processed, its 8-level metrics are passed to the Viterbi detector acting on its corresponding track. This means for each track, history for four of its states must be saved in addition to 4 of its next state metrics. The latency is therefore about the size of a whole sector. This means the whole sector is needed before we start getting outputs.

\section{RESUlts}

\section{A. Bit Error Rate Performance}

The results presented in Fig. 2 to Fig. 4 show Bit Error Rate (BER) performances of the two implemented SOVA/Viterbi concatenations. Fig. 2 presents a case with low ITI of $[0.25,1.0,0.25]$. Fig. 3 presents medium ITI of $[0.5,1.0,0.5]$, while Fig. 4 presents a case with high ITI of $[0.75,1.0,0.75]$.

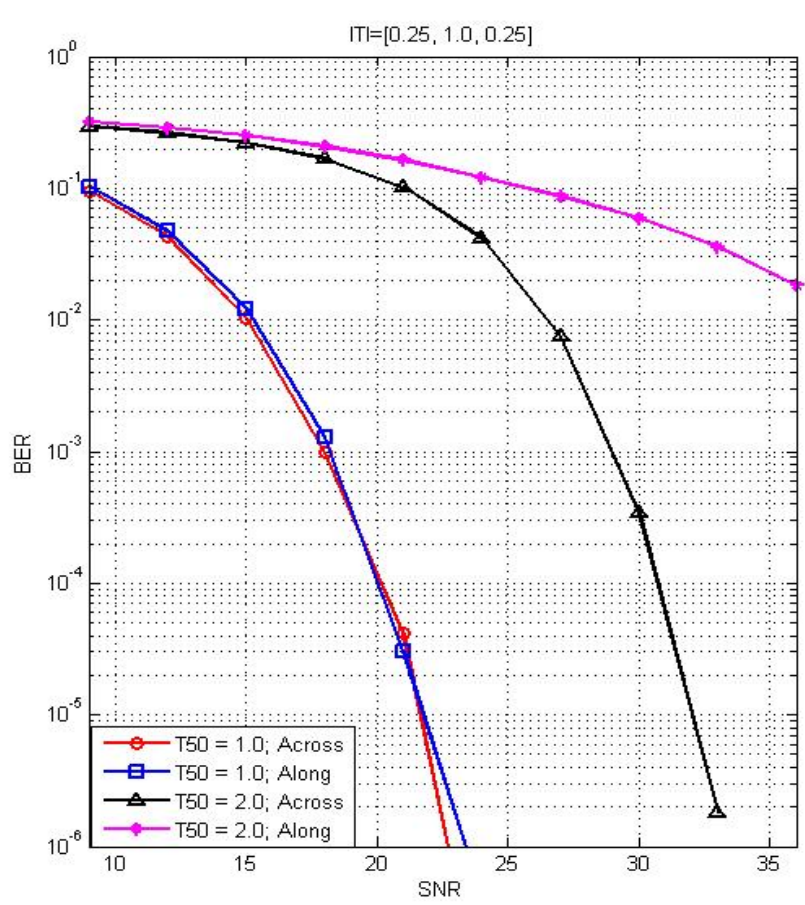

Fig. 2. 2D SOVA for $I T I=[0.25,1.00,0.25]$.

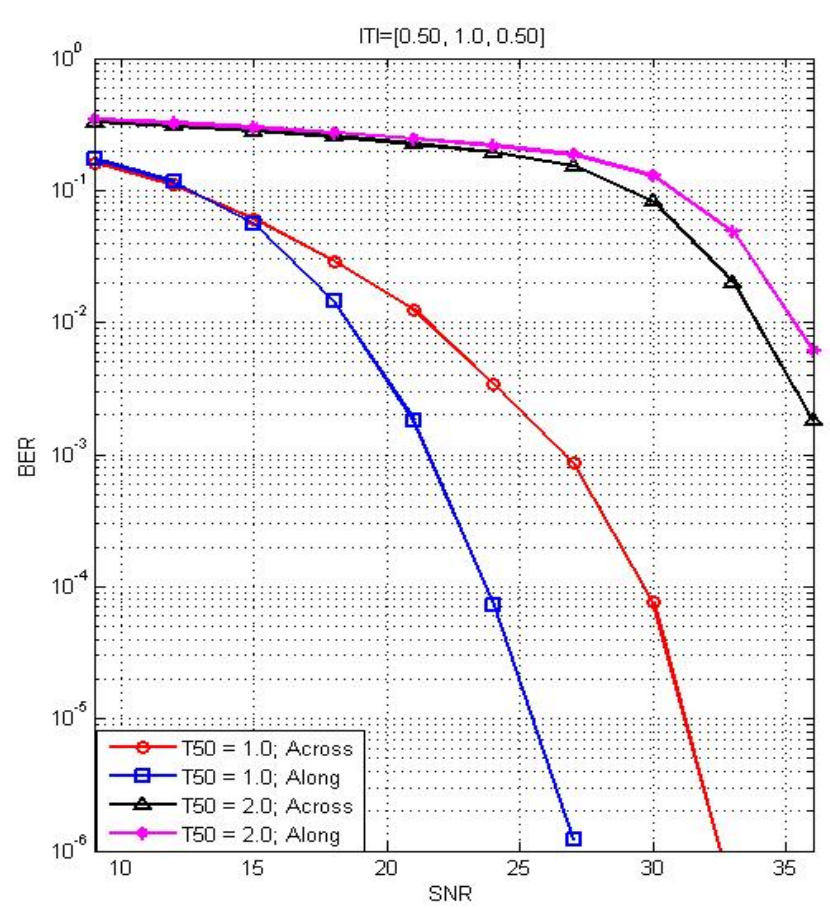

Fig. 3. 2D SOVA for $I T I=[0.50,1.00,0.50]$. 


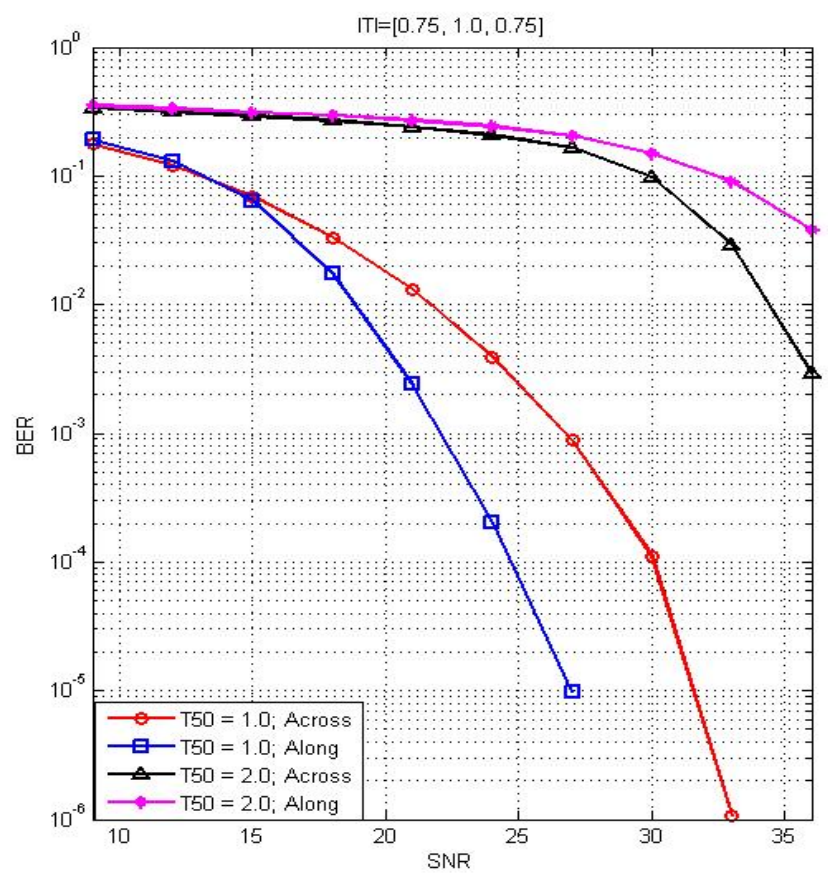

Fig. 4. 2D SOVA for $I T I=[0.75,1.00,0.75]$.

In the figures, it can be observed that for low density along-track $\left(T_{50}=1.0\right)$, the scheme that uses 2D-SOVA along track generally has a better performance, with the performance becoming clearer when there is high ITI. A performance gain of around $5 \mathrm{~dB}$ at $B E R=10^{-5}$ is obtained for high ITI.

For a situation with high density along track $\left(T_{50}=2.0\right)$, the use of 2D-SOVA across track provides a better performance. A very large gain is obtained for low ITI and very high ITI.

\section{B. Computational Complexity}

In this section " $\mathrm{A}$ " is used as a unit to indicate the number of additions, " $\mathrm{M}$ " is the unit of multiplications while " $\mathrm{C}$ " is the unit of the number of comparisons.

In both implementations, a shaping equaliser of length 13 is used for shaping. This means 13 multiplications and 12 additions are needed for shaping.

Both detectors do at most a total of 19 multiplications and additions (Maximum of $19 \mathrm{DMs}$ ) to determine distance metrics of each symbol using $D M=C_{n}-X_{n} * Y_{t}$.

The term $C_{n}$ is a precomputed one for each reference symbol " $X_{n}$ ", while $Y_{t}$ is the received signal.

To determine BM, a previous SM is added to appropriate DM. That is 512 (branches) additions in our case.

For each state, 7 comparisons are made to find the minimum BM (total of 448C). For 2D SOVA along track, with a trace back period of one unit (starting from the best state), 63 comparisons of the SM are done per cycle. For 2D SOVA across track, only 8 possible terminating states are compared (7C) after every eight symbols are processed across track.

The 8 metrics passed as DMs to the Viterbi detector are added to the appropriate (4) SMs of the Viterbi detector to get its BMs (8A). Each pair of BM is compared for each state to get the next SM (4C for the four states). In 2D SOVA along track, one additional comparison is done only at the end of each band of 8 track to pick from one of the 2 possible terminating states; while in 2D SOVA across track, the 4 states SMs are compared (3 comparisons) every cycle to determine the best state to start traceback from alongtrack.

Table 1 shows the summary of the complexity per bit processed (without simplification). The computational complexities are basically the same as can be seen.

TABLE 1: COMPUTATIONAL COMPLEXITIES.

\begin{tabular}{|l|l|l|}
\hline & Along & Across \\
\hline Equaliser & $12 \mathrm{~A}, 13 \mathrm{M}$, 0C & 12A, 13M, 0C \\
\hline 2D-SOVA & $531 \mathrm{~A}, 19 \mathrm{M}, 511 \mathrm{C}$ & $531 \mathrm{~A}, 19 \mathrm{M}, 449 \mathrm{C}$ \\
\hline Viterbi & $8 \mathrm{~A}, 0 \mathrm{M}$, 4C & $8 \mathrm{~A}, 0 \mathrm{M}$, 7C \\
\hline Total & 551A, 32M, 515C & 551A, 32M, 456C \\
\hline
\end{tabular}

\section{CONCLUSION}

From the results presented it is shown that if 2D SOVA is to be serially concatenated with the Viterbi detector in SMR media, carrying out 2D SOVA across the track has a better performance than 2D SOVA along track for high density but with more latency. For low density, 2D SOVA along track performs better. In terms of computational complexity, there is just a slight difference between the two. Memory requirement of 2D SOVA along track is higher than that of 2D SOVA across track.

\section{REFERENCES}

[1] R. Wood, "The feasibility of magnetic recording at 1 terabit per square inch," in IEEE Transactions on Magnetics, vol. 36, no 1, Jan. 2000, pp. 36-42.

[2] Y. Shiroishi, K. Fukuda, I. Tagawa, S. Takenoiri, H. Tanaka and N. Yoshikawa, "Future options for HDD storage" in IEEE Transactions on Magnetics, vol. 45, no. 10, Oct. 2009.

[3] R. L. White, R. M. H. New, and R. F. W. Pease, "Patterned media: A viable route to $50 \mathrm{Gbit} / \mathrm{in} 2$ and up for magnetic recording," IEEE Transactions on Magnetics, vol. 33, no. 1, Jan. 1997, pp. 990-995.

[4] R. Wood, M. Williams, A. Kavcic, and J. Miles, "The feasibility of magnetic recording at 10 terabits per square inch on conventional media," IEEE Transactions on Magnetics, vol. 45, no. 2, Feb, 2009, pp. 917-923.

[5] P. Shah, M.Z. Ahmed and Y. Kurihara, "New method for generalised PR target design for perpendicular magnetic recording," The Eighth Perpendicular Magnetic Recording Conference (PMRC2007), Oct. 15-17, 2007

[6] M. B. Abdulrazaq, M. Z. Ahmed, P. Davey, "Two dimensional equalisation of shingled write disk," Presented at International Conference on Magnetics, ICM2015, Jul. 2015.

[7] N. Zheng, K. S. Venkataraman, A. Kavcic, and T. Zhang, "A study of multitrack joint 2-D signal detection performance and implementation cost for shingled magnetic recording," IEEE Transactions on Magnetics, vol. 50, no. 6, Jun. 2014.

[8] M. B. Abdulrazaq, M. Z. Ahmed, P. Davey, "Concatenated 2D SOVA for two dimensional maximum likelihood detection," Presented at Telecommunications Forum Telfor (TELFOR2015), Nov. 24-26, 2015.

[9] N. Zheng and T. Zhang, "Design of Low-Complexity 2-D SOVA detector for shingled magnetic recording," IEEE Transactions on Magnetics, vol. 51, no. 4, Apr. 2015.

[10] W. Zheng, "Channel modeling, signal processing and coding for perpendicular magnetic recording," $\mathrm{PhD}$ dissertation, Electrical Engineering (Communication Theory and Systems), University of California, San Diego, 2009.

[11] N. Awad, "On reducing the decoding complexity of shingled magnetic recording system," $\mathrm{PhD}$ dissertation, Plymouth University, UK, May. 2013. 